\title{
Ranking Tehran's Stock Exchange Top Fifty Stocks Using Fundamental Indexes and Fuzzy TOPSIS
}

\author{
Ali Mohammad Kimiagari \\ Dpt of Industrial Engineering \& Management Systems \\ Amir Kabir University of Technology \\ (Tehran Polytechnic) \\ Tehran, Iran
}

\begin{abstract}
Investment through the purchase of securities, constitute an important part of countries economic exchange. Therefore, making decisions about investing in a particular stock has become one of the most controversial areas of economic and financial research and various institutions have began to rank companies stock and determine priorities of stock purchase to investment. The current research, with the determination of important required indexes for companies ranking based on their shares value on the Tehran stock exchange, can greatly help to the accurate ranking of fifty premier listed companies. Initial ranking indicators are extracted and then a decision-making group (exchange experts) with the use of the Delphi method and also non-parametric statistic methods, determines the final indexes. Then, by using Fuzzy ANP, weight criteria are obtained with taking into account their interaction with each other. Finally, using fuzzy TOPSIS and information extraction about the premier fifty listed companies of Tehran stock exchange in $\mathbf{2 0 1 4}$ are ranked with the software "Rahavard Novin". Sensitivity analysis to criteria weight and relevant analysis presentation was conducted at the end of the study procedures.
\end{abstract}

Keywords-performance evaluation; stock efficiency; companies ranking; ANP Fuzzy; TOPSIS.

\section{INTRODUCTION}

Much research in ranking and evaluating companies has been conducted, especially on stock exchange companies. In [1], authors applied time series analysis and multi-criteria decision to anticipate the financial performance of companies. The aim of this study was to estimate the financial performance of companies in Turkey between 2012 and 2015. For this purpose, two time series analysis and multi-criteria decision approach analysis were used. In [2], authors checked to evaluate the efficacy data on the value of stock options in the Taiwan market. In [3], authors began to combine soft computing and fundamental analysis to select stocks. The results show that the proposed method could pay to stock selection, well. In [4], using CBR select stocks was investigated. The study results show that the proposed model could select the stock's choice based on the determined indexes very well. In [5], the performance of banks was evaluated with fuzzy hierarchical analysis and fuzzy TOPSIS. In [6], authors surveyed the TOPSIS approach as a ranking approach.

\author{
Elham Sabeti Saleh \\ Dpt of Industrial Engineering \& Management Systems \\ Amir Kabir University of Technology \\ (Tehran Polytechnic) \\ Tehran, Iran
}

In this paper, the 2014 Tehran Stock Exchange market is considered and its statistical population is the heads of 87 Tehran Stock Exchange agencies. Also for the financial information extraction of the companies, the "Rahavard Novin" software is used. Two fuzzy network analysis and Fuzzy TOPSIS technique are used to analyze the results and rank companies. Two matrix (middle number and near fuzzy number) of each matrix are derived and then an adaption of each matrix based on the computing hours is computed.

\section{BASICS OF USED ANALYSIS METHODS}

The steps of computing fuzzy matrix pairwise comparison compatibility rate are:

\section{A. Step 1}

In the first phase divide triangular matrix to two matrixes.

First matrix is formed of middle numbers of triangular inferences $A^{m}=\left[a_{i j m}\right]$ and the second matrix contains Geometric mean of the upper and lower triangular numbers,

$$
A^{g}=\sqrt{a_{i j u} \cdot a_{i j l}} \text {. }
$$

\section{B. Step 2}

Each vector weight vector with using saati method is computed as below:

$$
\begin{gathered}
w_{i}^{m}=\frac{1}{n} \sum_{j=1}^{n} \frac{a_{i j m}}{\sum_{i=1}^{n} a_{i j m}} \\
\text { Which in it } w^{m}=\left[w_{i}^{m}\right] \\
w_{i}^{g}=\frac{1}{n} \sum_{j=1}^{n} \frac{\sqrt{a_{i j u} \cdot a_{i j l}}}{\sum_{i=1}^{n} \sqrt{a_{i j u} \cdot a_{i j l}}} \\
\text { Which in it } w^{g}=\left[w_{i}^{g}\right]
\end{gathered}
$$

\section{Step 3}

The biggest Eigen vector for each matrix is calculated with below relations: 


$$
\begin{gathered}
\lambda_{\max }^{m}=\frac{1}{n} \sum_{i=1}^{n} \sum_{j=1}^{n} a_{i j m}\left(\frac{w_{j}^{m}}{w_{i}^{m}}\right) \\
\lambda_{\max }^{g}=\frac{1}{n} \sum_{i=1}^{n} \sum_{j=1}^{n} \sqrt{a_{i j u} \cdot a_{i j l}}\left(\frac{w_{j}^{g}}{w_{i}^{g}}\right)
\end{gathered}
$$

\section{Step 4}

Consistency index is calculated with using below relations.

$$
\begin{aligned}
C I^{m} & =\frac{\left(\lambda_{\max }^{m}-n\right)}{(n-1)} \\
C I^{g} & =\frac{\left(\lambda_{\max }^{g}-n\right)}{(n-1)}
\end{aligned}
$$

\section{E. Step5}

To calculate the consistency rate (CR), the CI index is divided to random index (RI). If the result value is less than 0.1 , matrix diagnosed consistent and usable. To produce random matrixes, first the middle value of triangular fuzzy number produced randomly in $[1 / 9,9]$ interval mutually. Then, low bound of each triangular number in [produced middle value, 1/9] interval and upper bound value in [1/9, produced middle value] interval is produced randomly and at last, their random index value was obtained by dividing resultant random matrix to two middle bound matrix and geometric mean of up and low bounds matrix. We compare computed consistency rate for two matrix based on below relations with threshold 0.1 :

$$
\begin{aligned}
& C R^{g}=\frac{C I^{g}}{R I^{g}} \\
& C R^{m}=\frac{C I^{m}}{R I^{m}}
\end{aligned}
$$

If both indexes were lower than 0.1 , fuzzy matrix is consistence. While both were more than 0.1 , decision-making will be asked to reconsider the priorities presented and while only $C R^{m}\left(C R^{g}\right)$ was more than 0.1 , decision-making of reconsidering in middle values (bounds) make fuzzy inference.

\section{STEPS OF OBTAINING COMPONENT WEIGHTS WITH FUZZY NETWORK ANALYSIS}

Based on super-matrix, steps of component weights computing include:

The first step is to bring together experts comments, the paired comparisons of geometric mean respondents is taken.

Second: the Eigen vector comparison: to calculate Eigen vector comparison each pairwise comparisons tables aggregated, according to equation logarithmic least squares method is used.

$$
w_{k}^{s}=\frac{\left(\prod_{j=1}^{n} a_{k j}^{s}\right)^{1 / n}}{\sum_{i=1}^{n}\left(\prod_{j=1}^{n} a_{i j}^{m}\right)^{1 / n}}, \quad s \in\{l, m, u\}
$$

As:

$$
\widetilde{w}_{k}=\left(w_{k}^{l}, w_{k}^{m}, w_{k}^{u}\right) \quad k=1,2,3, \ldots, n
$$

Third: forming Eigen vector matrixes $\left(W_{i j}\right)$ : these matrixes include Eigen vectors which obtained from second step of pairwis comparisons.

Fourth step: computing final levels weights: for computing final weight of each level component $\left(W_{i}^{*}\right)$ the multiplication of Eigen matrix-vector of inner relation in same level of Eigen vector must be multiplied by the higher level of upper level.

$$
W_{i}^{*}=W_{i i} \times W_{i(i-1)} \times W_{i-1}^{*}
$$

While, there was not one level ${ }^{W_{i i}}$ matrix , it is necessary to substitute identity matrix with the same degree of that matrix. In the other word, below formula is used.

$$
W_{i}^{*}=I \times W_{i(i-1)} \times W_{i-1}^{*}
$$

TOPSIS method is a common approach in decision making multi-criteria (MADM) to the development phase space. This method requires a decision-making matrix which rows of this matrix is options and columns of the matrix are the criteria [7, $8,9]$. With a systematic approach, we can develop a TOPSIS decision making method to fuzzy space. To increase the accuracy of calculations, we can assume that the degree of importance of decision criteria and qualitative variables rates as linguistic variables were collected. In fuzzy space, linguistic variables can be defined in the form of fuzzy numbers. Many decision-making techniques in fuzzy space rather profit paired comparisons to reach a decision matrix. Always a multi-criteria decision-making problem in fuzzy space can be shown in matrix form as below.

$$
\begin{gathered}
\tilde{D}=\left[\begin{array}{cccc}
\tilde{x}_{11} & \tilde{x}_{12} & \ldots & \tilde{x}_{1 n} \\
\tilde{x}_{21} & \tilde{x}_{22} & \ldots & \tilde{x}_{2 n} \\
\cdot & \cdot & & \cdot \\
\cdot & \cdot & \ldots & \cdot \\
\cdot & \cdot & & \cdot \\
\tilde{x}_{m 1} & \tilde{x}_{m 2} & \ldots & \tilde{x}_{m n}
\end{array}\right] \\
\tilde{W}=\left[\tilde{w}_{1}, \tilde{w}_{2}, \ldots, \tilde{w}_{n}\right]
\end{gathered}
$$

Note that each of the linguistic variables $X_{i j}$ and $W_{j}$ can be in the form of triangular fuzzy numbers is defined as follows:

$$
\begin{gathered}
\tilde{X}_{i j}=\left(a_{i j}, b_{i j}, c_{i j}\right) \\
\tilde{W}_{j}=\left(w_{j 1}, w_{j 2}, w_{j 3}\right)
\end{gathered}
$$

For the TOPSIS technique, it is necessary to normalize obtained data in decision-making matrix using Euclidean norms. This normalization method has high complexity. But if we want to use this technique in a fuzzy space, we can use simpler norms such as linear to data homogenization. Thus, normalized fuzzy decision matrix is obtained as follows 


$$
\tilde{R}=\left[\tilde{r}_{i j}\right]_{m \times n}
$$

If we define $\mathrm{B}$ and $\mathrm{C}$ respectively as set of same direction criteria with cost and profit, we have:

$$
\begin{gathered}
\tilde{r}_{i j}=\left(\frac{a_{i j}}{c_{j}^{*}}, \frac{b_{i j}}{c_{j}^{*}}, \frac{c_{i j}}{c_{j}^{*}}\right) \quad j \in B \\
\tilde{r}_{i j}=\left(\frac{a_{j}^{-}}{c_{i j}}, \frac{a_{j}^{-}}{b_{i j}}, \frac{c a_{j}^{-}}{c_{i j}}\right) \quad j \in C \\
c_{j}^{*}=\max \quad c_{i j} \quad \text { if } \quad j \in B \\
a_{j}^{-}=\min \quad a_{i j} \quad \text { if } \quad j \in C
\end{gathered}
$$

By using this normalizing method, we can now homogenize all fuzzy data based on obtained triangular numbers in a range between zero and one. Now, based on row vector of criteria weights (W) and normalized decision matrix R can be obtained the decision weighted matrix.

$$
\tilde{V}=\left[\tilde{v}_{i j}\right]_{m \times n}
$$

So that each of the elements of this matrix are equal:

$$
\tilde{v}_{i j}=\tilde{r}_{i j} \cdot \tilde{w}_{j} \quad \mathrm{i}=1,2, \ldots, \mathrm{m}, \quad \mathrm{j}=1,2, \ldots, \mathrm{n},
$$

Each of the weighted normalized decision matrix elements are fuzzy triangular numbers which their numbers are homogenized between zero and one. Based on this matrix, positive and negative ideal options can be defined:

$$
\begin{aligned}
& A^{+}=\left(\tilde{v}_{1}^{*}, \tilde{v}_{2}^{*}, \ldots, \tilde{v}_{n}^{*}\right) \\
& A^{-}=\left(\tilde{v}_{1}^{-}, \tilde{v}_{2}^{-}, \ldots, \tilde{v}_{n}\right)
\end{aligned}
$$

Where:

$$
\begin{aligned}
& \tilde{v}_{j}^{*}=(1,1,1) \\
& \tilde{v}_{j}=(0,0,0)
\end{aligned}
$$

After calculating positive and negative ideal options, the distance of each options of these two ideal choices will be achieved

$$
\begin{array}{ll}
d_{i}^{*}=\sum_{j=1}^{n} d\left(\tilde{v}_{i j}-\tilde{v}_{j}^{*}\right) & i=1,2, \ldots, m \\
d_{i}^{-}=\sum_{j=1}^{n} d\left(\tilde{v}_{i j}-\tilde{v}_{j}^{-}\right) & i=1,2, \ldots, m
\end{array}
$$

At last, the closeness coefficient is calculated to determine the priorities of options. This coefficient can be obtained based on the values $d_{i}^{*}, d_{i}^{-}$of each alternative as follows:

$$
C C_{i}=\frac{d_{i}^{-}}{d_{i}^{*}+d_{i}^{-}} \quad i=1,2, \ldots, m
$$

Now, we can sort all options based on higher value of $C C_{i}$.

\section{DATA ANALYSIS RESULTS}

In this step, the most important financial ratios were identified as primary indexes. These indexes are mentioned in 12 criteria-groups in below and were offered to experts by questionnaire to each experts mention their opinions about importance of this criterion in the ranking process of Tehran Stock Exchange company's shares (Table I). Answers of 51

\begin{tabular}{|c|c|c|c|c|c|c|}
\hline $\begin{array}{l}\text { Prioritizing } \\
\text { indicators }\end{array}$ & Beta coefficient & $\begin{array}{l}\text { Earnings per share } \\
\text { (EPS) }\end{array}$ & Sales growth & $\begin{array}{l}\text { Price to earnings } \\
\text { ratio } P / E\end{array}$ & $\begin{array}{l}\text { Dividend per share } \\
\text { (DPS) }\end{array}$ & $\begin{array}{l}\text { Growth in profit } \\
\text { margins }\end{array}$ \\
\hline Beta coefficient & $(1,1,1)$ & $(4,5.13,6.22)$ & $(2,2.47,2.89)$ & $(6,7,8)$ & $(3.64,4.72,5.77)$ & $(4.58,5.6,6.61)$ \\
\hline $\begin{array}{l}\text { Earnings per share } \\
\text { (EPS) }\end{array}$ & $(0.17,0.2,0.25)$ & $(1,1,1)$ & $(0.33,0.41,0.5)$ & $(0.8,1,1.36)$ & $(1.59,2.03,2.63)$ & $(1.59,2.09,2.72)$ \\
\hline Sales growth & $(0.35,0.41,0.5)$ & $(2,2.47,3.11)$ & $(1,1,1)$ & $(4,5.13,6.22)$ & $(2.29,2.76,3.42)$ & $(6.35,7.4,8.44)$ \\
\hline $\begin{array}{l}\text { Price to earnings } \\
\text { ratio } \mathrm{P} / \mathrm{E}\end{array}$ & $(0.13,0.15,0.17)$ & $(0.74,1,1.26)$ & $(0.17,0.2,0.25)$ & $(1,1,1)$ & $(0.7,0.85,1)$ & $(0.63,0.7,0.8)$ \\
\hline $\begin{array}{l}\text { Dividend per share } \\
\text { (DPS) }\end{array}$ & $(0.18,0.22,0.28)$ & $(0.39,0.5,0.63)$ & $(0.3,0.37,0.44)$ & $(1,1.19,1.45)$ & $(1,1,1)$ & $(1,1.12,1.26)$ \\
\hline $\begin{array}{l}\text { Growth in profit } \\
\text { margins }\end{array}$ & $(0.16,0.18,0.22)$ & $(0.37,0.49,0.63)$ & $(0.12,0.14,0.16)$ & $(1.26,1.45,1.59)$ & $(0.8,0.9,1)$ & $(1,1,1)$ \\
\hline
\end{tabular}
completed questionnaires regarding the importance of criteria of ranking Tehran Stock Exchange company shares is shown as table and graph with Delphi method by descriptive approach. In this questionnaire, the importance of intended indexes in the direction of the research purposes has been questioned. In this part, with considering Figure 1, we calculate each fifty premier company ranking indexes weight and active company of Tehran Stock Exchange with ANP fuzzy approach for group decision making.

TABLE I. THE IDENTIFIED PRIMARY CRITERIA FOR RANKING COMPANY STOCKS

\begin{tabular}{|c|c|c|c|}
\hline Row & Index & Row & Index \\
\hline 1 & Beta Coefficient (systematic risk) & 7 & Returns \\
\hline 2 & $\begin{array}{c}\text { Financial ratios (liquidity, } \\
\text { efficiency, etc.) }\end{array}$ & 8 & Risk and Return \\
\hline 3 & Dividend Per Share (DPS) & 9 & $\begin{array}{c}\text { Earnings per share } \\
\text { (EPS) }\end{array}$ \\
\hline 4 & Capital increase & 10 & Sales growth \\
\hline 5 & Balance sheet & 11 & $\begin{array}{c}\text { Growth in profit } \\
\text { margins }\end{array}$ \\
\hline 6 & Profit and loss & 12 & $\begin{array}{c}\text { Price to earnings ratio } \\
\text { (P/E) }\end{array}$ \\
\hline
\end{tabular}

TABLE II. COMMON PAIRED COMPARISONS AVERAGE FUZZY IN RANKING INDICATORS OF ACTIVE COMPANIES AND TOP STOCKS (MATRIX) 


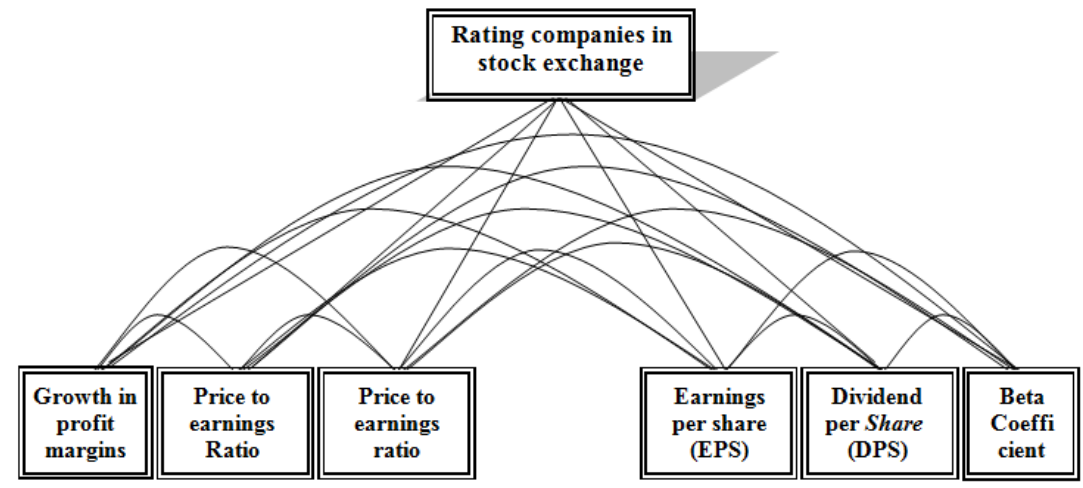

Fig. 1. Company ranking indexes weight and active company of Tehran Stock Exchange with ANP fuzzy

Then, pairwise comparison of 6 final criteria is defined, rather than goal and other criteria (control criteria) including 7 tables in second questionnaire were offered to persons who complete the first questionnaire and decision- making group members compared criteria pairwise comparison in Verbal terms forms "same preferences, small preferred, the strong preference, preferred very strong "which respectively represent fuzzy numbers are $1,3,5,7,9$. It should be noted that the decision group members were asked to fill out entries above the main diagonal of pair-wise comparison tables, because in considered fuzzy ANP approach, pairwise comparison matrices elements are reversed in comparison to the main diameter. Summarizing shows the pairwise comparison of indexes for $^{W}$ matrix (Table II-VIII).

TABLE III. COMMON PAIRED COMPARISONS AVERAGE FUZZY IN RANKING INDICATORS IN RELATION TO BETA COEFFICIENT INDEX

\begin{tabular}{|c|c|c|c|c|c|}
\hline Beta coefficient & $\begin{array}{l}\text { Earnings per share } \\
\text { (EPS) }\end{array}$ & Sales growth & $\begin{array}{l}\text { Price to earnings ratio } \\
\text { P/E }\end{array}$ & $\begin{array}{c}\text { Dividend per share } \\
\text { (DPS) }\end{array}$ & $\begin{array}{c}\text { Growth in profit } \\
\text { margins }\end{array}$ \\
\hline $\begin{array}{c}\text { Earnings per share } \\
\text { (EPS) }\end{array}$ & $(1,1,1)$ & $(0.33,0.41,0.5)$ & $(0.56,0.64,0.75)$ & $(1,1.19,1.45)$ & $(1.48,1.71,1.96)$ \\
\hline Sales growth & $(2,2.47,3.11)$ & $(1,1,1)$ & $(5.25,6.26,7.27)$ & $(1.82,2.27,2.89)$ & $(0.61,0.72,0.86)$ \\
\hline $\begin{array}{l}\text { Price to earnings ratio } \\
\mathrm{P} / \mathrm{E}\end{array}$ & $(1.34,1.58,1.82)$ & $(0.14,0.16,0.2)$ & $(1,1,1)$ & $(1.34,1.58,1.82)$ & $(0.18,0.22,0.28)$ \\
\hline $\begin{array}{c}\text { Dividend per share } \\
\text { (DPS) }\end{array}$ & $(0.7,0.85,1)$ & $(0.35,0.45,0.56)$ & $(0.56,0.64,0.75)$ & $(1,1,1)$ & $(0.56,0.64,0.75)$ \\
\hline $\begin{array}{c}\text { Growth in profit } \\
\text { margins }\end{array}$ & $(0.52,0.59,0.68)$ & $(1.17,1.41,1.66)$ & $(3.64,4.72,5.77)$ & $(1.34,1.58,1.82)$ & $(1,1,1)$ \\
\hline
\end{tabular}

TABLE IV. COMMON PAIRED COMPARISONS AVERAGE FUZZY IN RANKING INDICATORS IN RELATION TO EARNINGS PER SHARE (EPS)

\begin{tabular}{|c|c|c|c|c|c|}
\hline $\begin{array}{c}\text { Earnings per share } \\
\text { (EPS) }\end{array}$ & Beta coefficient & Sales growth & $\begin{array}{l}\text { Price to earnings ratio } \\
\mathrm{P} / \mathrm{E}\end{array}$ & $\begin{array}{c}\text { Dividend per share } \\
\text { (DPS) }\end{array}$ & $\begin{array}{c}\text { Growth in profit } \\
\text { margins }\end{array}$ \\
\hline Beta coefficient & $(1,1,1)$ & $(0.71,0.85,1)$ & $(1.42,1.74,2.24)$ & $(4,5,6)$ & $(1.16,1.35,1.59)$ \\
\hline Sales growth & $(1,1.19,1.42)$ & $(1,1,1)$ & $(2,3,4)$ & $(0.71,0.85,1)$ & $(0.87,1,1.16)$ \\
\hline $\begin{array}{l}\text { Price to earnings ratio } \\
\mathrm{P} / \mathrm{E}\end{array}$ & $(0.45,0.58,0.71)$ & $(0.25,0.34,0.5)$ & $(1,1,1)$ & $(0.82,1,1.23)$ & $(0.82,1,1.23)$ \\
\hline $\begin{array}{c}\text { Dividend per share } \\
\text { (DPS) }\end{array}$ & $(0.17,0.2,0.25)$ & $(1,1.19,1.42)$ & $(0.82,1,1.23)$ & $(1,1,1)$ & $(0.87,1,1.16)$ \\
\hline $\begin{array}{c}\text { Growth in profit } \\
\text { margins }\end{array}$ & $(0.64,0.75,0.87)$ & $(0.87,1,1.16)$ & $(0.82,1,1.23)$ & $(0.87,1,1.16)$ & $(1,1,1)$ \\
\hline
\end{tabular}

TABLE V. COMMON PAIRED COMPARISONS AVERAGE FUZZY IN RANKING INDICATORS IN RELATION TO SALES GROWTH

\begin{tabular}{|c|c|c|c|c|c|}
\hline Sales growth & Beta coefficient & $\begin{array}{c}\text { Earnings per share } \\
\text { (EPS) }\end{array}$ & $\begin{array}{c}\text { Price to earnings ratio } \\
\mathbf{P} / \mathbf{E}\end{array}$ & $\begin{array}{c}\text { Dividend per share } \\
\text { (DPS) }\end{array}$ & $\begin{array}{c}\text { Growth in profit } \\
\text { margins }\end{array}$ \\
\hline Beta coefficient & $(1,1,1)$ & $(0.45,0.58,0.71)$ & $(6.93,7.94,8.95)$ & $(4.9, \quad 5.92,6.93)$ & $(1,1.19,1.42)$ \\
\hline $\begin{array}{c}\text { Earnings per share } \\
\text { (EPS) }\end{array}$ & $(1.42,1.74,2.24)$ & $(1,1,1)$ & $(8,9,10)$ & $(4,5.2,6.33)$ & $(4,5,6)$ \\
\hline $\begin{array}{c}\text { Price to earnings ratio } \\
\mathbf{P} / \mathbf{E}\end{array}$ & $(0.12,0.13,0.15)$ & $(0.1,0.12,0.13)$ & $(1,1,1)$ & $\left(\begin{array}{lll}0.36, & 0.38, & 0.41)\end{array}\right.$ & $(0.12,0.13,0.15)$ \\
\hline $\begin{array}{c}\text { Dividend per share } \\
\text { (DPS) }\end{array}$ & $(0.15,0.17,0.21)$ & $(0.16, \quad 0.2 ， 0.25)$ & $(2.45,2.65,2.83)$ & $(1,1,1)$ & $(0.13,0.15,0.18)$ \\
\hline $\begin{array}{c}\text { Growth in profit } \\
\text { margins }\end{array}$ & $(0.71,0.85,1)$ & $(0.17, \quad 0.2 ， 0.25)$ & $(6.93,7.94,8.95)$ & $(5.66,6.71,7.75)$ & $(1,1,1)$ \\
\hline
\end{tabular}


TABLE VI. COMMON PAIRED COMPARISONS AVERAGE FUZZY IN RANKING INDICATORS IN RELATION TO PRICE TO EARNINGS RATIO P/E

\begin{tabular}{|c|c|c|c|c|c|}
\hline $\begin{array}{l}\text { Price to earnings ratio } \\
\mathrm{P} / \mathrm{E}\end{array}$ & Beta coefficient & $\begin{array}{c}\text { Earnings per share } \\
\text { (EPS) }\end{array}$ & Sales growth & $\begin{array}{c}\text { Dividend per share } \\
\text { (DPS) }\end{array}$ & $\begin{array}{c}\text { Growth in profit } \\
\text { margins }\end{array}$ \\
\hline Beta coefficient & $(1,1,1)$ & $(2.45,2.65,2.83)$ & $(2.45,2.65,2.83)$ & $(1.23,1.53,2)$ & $(2.83,3.88,4.9)$ \\
\hline $\begin{array}{c}\text { Earnings per share } \\
\text { (EPS) }\end{array}$ & $(0.36,0.38,0.41)$ & $(1,1,1)$ & $(1.42,1.74,2)$ & $(1.42,1.74,2)$ & $(0.5,0.66,0.82)$ \\
\hline Sales growth & $(0.36,0.38,0.41)$ & $(0.5,0.58,0.71)$ & $(1,1,1)$ & $(0.41,0.45,0.5)$ & $(0.5,0.66,0.82)$ \\
\hline $\begin{array}{c}\text { Dividend per share } \\
\text { (DPS) }\end{array}$ & $(0.5,0.66,0.82)$ & $(0.5,0.58,0.71)$ & $(2,2.24,2.45)$ & $(1,1,1)$ & $(0.58,0.78,1)$ \\
\hline $\begin{array}{c}\text { Growth in profit } \\
\text { margins }\end{array}$ & $(0.36,0.26,0.21)$ & $(1.23,1.53,2)$ & $(1.23,1.53,2)$ & $(1,1.3,1.74)$ & $(1,1,1)$ \\
\hline
\end{tabular}

TABLE VII. COMMON PAIRED COMPARISONS AVERAGE FUZZY IN RANKING INDICATORS IN RELATION TO DIVIDEND PER SHARE (DPS)

\begin{tabular}{|c|c|c|c|c|c|}
\hline $\begin{array}{c}\text { Dividend per share } \\
\text { (DPS) }\end{array}$ & Beta coefficient & $\begin{array}{c}\text { Earnings per share } \\
\text { (EPS) }\end{array}$ & Sales growth & $\begin{array}{l}\text { Price to earnings ratio } \\
\mathbf{P} / \mathbf{E}\end{array}$ & $\begin{array}{c}\text { Growth in profit } \\
\text { margins }\end{array}$ \\
\hline Beta coefficient & $(1,1,1)$ & $(2,2.24,2.45)$ & $(4,5,6)$ & $(1,1.3,1.74)$ & $(0.82,1,1.23)$ \\
\hline $\begin{array}{c}\text { Earnings per share } \\
\text { (EPS) }\end{array}$ & $\left(\begin{array}{lll}0.41, & 0.45, & 0.5\end{array}\right)$ & $(1,1,1)$ & $(1.42,1.74,2)$ & $(0.21, \quad 0.26,0.36)$ & $(0.5, \quad 0.66,0.82)$ \\
\hline Sales growth & $(0.17,0.2,0.25)$ & $(0.5,0.58,0.71)$ & $(1,1,1)$ & $(0.15,0.17,0.21)$ & $(0.5,0.66,0.82)$ \\
\hline $\begin{array}{l}\text { Price to earnings ratio } \\
\mathrm{P} / \mathrm{E}\end{array}$ & $(0.58,0.78,1)$ & $(2.83,3.88,4.9)$ & $(4.9, \quad 5.92,6.93)$ & $(1,1,1)$ & $(1.16,1.35,1.59)$ \\
\hline $\begin{array}{c}\text { Growth in profit } \\
\text { margins }\end{array}$ & $(0.82,1,1.23)$ & $(1.23,1.53,2)$ & $(1.23,1.53,2)$ & $(0.64,0.75,0.87)$ & $(1,1,1)$ \\
\hline
\end{tabular}

TABLE VIII. COMMON PAIRED COMPARISONS AVERAGE FUZZY IN RANKING INDICATORS IN RELATION TO GROWTH IN PROFIT MARGINS

\begin{tabular}{|c|c|c|c|c|c|}
\hline $\begin{array}{c}\text { Growth in profit } \\
\text { margins }\end{array}$ & Beta coefficient & $\begin{array}{c}\text { Earnings per share } \\
\text { (EPS) }\end{array}$ & $\begin{array}{c}\text { Sales growth } \\
\text { Price to earnings ratio } \\
\text { P/E }\end{array}$ & $\begin{array}{c}\text { Dividend per share } \\
\text { (DPS) }\end{array}$ \\
\hline Beta coefficient & $(1,1,1)$ & $(2,2.24,2.45)$ & $(0.71,1,1.42)$ & $(2.45,2.65,2.83)$ & $(1.23,1.53,2)$ \\
\hline $\begin{array}{c}\text { Earnings per share } \\
\text { (EPS) }\end{array}$ & $(0.41,0.45,0.5)$ & $(1,1,1)$ & $(0.21,0.26,0.36)$ & $(0.41,0.45,0.5)$ & $(0.25,0.34,0.5)$ \\
\hline Sales growth & $(0.71,1,1.42)$ & $(2.83,3.88,4.9)$ & $(1,1,1)$ & $(0.5,0.58,0.71)$ & $(2,2.24,2.45)$ \\
\hline $\begin{array}{c}\text { Price to earnings ratio } \\
\text { P/E }\end{array}$ & $(0.36,0.38,0.41)$ & $(2,2.24,2.45)$ & $(1.42,1.74,2)$ & $(1,1,1)$ \\
\hline $\begin{array}{c}\text { Dividend per share } \\
\text { (DPS) }\end{array}$ & $(0.5,0.66,0.82)$ & $(2,3,4)$ & $(0.41,0.45,0.5)$ & $(0.41,0.45,0.5)$ & $(1,1,1)$ \\
\hline
\end{tabular}

Fuzzy weight for each factor of prioritizing top and active companies in stock exchange is as shown in Table IX. The fuzzy weight of each prioritizing indicator of active and top companies in stock exchange is shown in Table X. In the next

phase, ranking of companies using TOPSIS fuzzy approach is determined with respect to the decision criteria specified above and the above weights (Tables XII-XII).

TABLE IX. FUZZY WEIGHT FOR EACH FACTOR OF PRIORITIZING TOP AND ACTIVE COMPANIES IN STOCK EXCHANGE

\begin{tabular}{|c|c|c|c|c|c|c|c|}
\hline \multirow[t]{2}{*}{ Prioritizing indicators } & \multicolumn{3}{|c|}{$\left(\prod_{i=1}^{6} a_{k j}^{s}\right)^{1 / 6} s \in\{l, m, u\}$} & \multicolumn{3}{|c|}{$w_{k}^{s}=\frac{(j=1)}{6(6))^{1 / 6}}$} & \multirow{2}{*}{ Weight of each indicator } \\
\hline & l & $\mathbf{m}$ & $\mathbf{u}$ & I & $\mathbf{m}$ & $\mathbf{u}$ & \\
\hline Beta coefficient & 0.967 & 1.338 & 1.849 & 0.114 & 0.158 & 0.218 & $(0.11,0.15,0.21)$ \\
\hline Earnings per share (EPS) & 1.355 & 1.870 & 2.533 & 0.160 & 0.221 & 0.299 & $(0.16,0.22,0.29)$ \\
\hline Sales growth & 1.154 & 1.621 & 2.227 & 0.136 & 0.191 & 0.263 & $(0.13,0.19,0.26)$ \\
\hline Price to earnings ratio $\mathrm{P} / \mathrm{E}$ & 0.659 & 0.909 & 1.251 & 0.078 & 0.107 & 0.148 & $(0.78,0.10,0.15)$ \\
\hline Dividend per share (DPS) & 0.677 & 0.940 & 1.281 & 0.080 & 0.111 & 0.151 & $(0.08,0.11,0.15)$ \\
\hline Growth in profit margins & 1.288 & 1.789 & 2.429 & 0.152 & 0.211 & 0.287 & $(0.15,0.21,0.28)$ \\
\hline$\sum_{i-1}^{6}\left(\prod_{j=1}^{6} a_{k j}^{m}\right)^{1 / 6}$ & & 8.467 & & & & & \\
\hline
\end{tabular}


TABLE X. FUZZY WEIGHT OF EACH PRIORITIZING INDICATORS

\begin{tabular}{|c|c|c|}
\hline Prioritizing indicators & Absolute Weight of indicators \\
\hline Beta coefficient & 0.161 \\
\hline Earnings per share (EPS) & 0.224 \\
\hline Sales growth & 0.194 & 0.109 \\
\hline Price to earnings ratio P/E & 0.113 & 3 \\
\hline Dividend per share (DPS) & 0.214 & \\
\hline Growth in profit margins & 5 & \\
\hline
\end{tabular}

TABLE XI. FIFTY PREMIER COMPANY DATA RANKING INDEXES

\begin{tabular}{|c|c|c|c|c|c|c|c|c|c|c|c|}
\hline $\begin{array}{c}\text { Growth in } \\
\text { profit margins }\end{array}$ & DPS & $\mathbf{P} / \mathbf{E}$ & $\begin{array}{c}\text { Sales } \\
\text { growth }\end{array}$ & EPS & $\begin{array}{c}\text { Beta } \\
\text { coefficient }\end{array}$ & $\begin{array}{l}\text { Growth } \\
\text { in profit } \\
\text { margins }\end{array}$ & DPS & $\mathbf{P} / \mathbf{E}$ & $\begin{array}{c}\text { Sales } \\
\text { growth }\end{array}$ & EPS & $\begin{array}{c}\text { Beta } \\
\text { coefficient }\end{array}$ \\
\hline 11.998 & 128.750 & 6.143 & 52.975 & 39.735 & 1.510 & 8.333 & 520.000 & 2.583 & 27.430 & 28.145 & 0.033 \\
\hline-3.888 & 127.000 & 3.363 & 13.735 & 29.613 & 0.670 & -0.515 & 668.750 & 2.483 & 21.105 & 20.655 & 0.320 \\
\hline 13.523 & 117.500 & 9.035 & 43.523 & 18.243 & 0.513 & -55.113 & 307.500 & 7.040 & 412.638 & 63.478 & 0.953 \\
\hline 1.213 & 605.750 & 6.888 & 22.023 & 21.830 & 0.580 & 5.040 & 1680.000 & 5.390 & 21.048 & 5.928 & 0.528 \\
\hline 1.240 & 205.000 & 6.077 & 67.127 & 73.353 & 0.438 & 3.583 & 781.250 & 7.630 & 14.310 & 13.655 & 0.798 \\
\hline 3.517 & 132.500 & 18.680 & -6.750 & -9.910 & -0.002 & 1.713 & 2375.000 & 4.615 & 12.698 & 16.963 & 1.295 \\
\hline-34.055 & 500.000 & 4.780 & 49.868 & 71.138 & 0.000 & 9.358 & 722.500 & 7.105 & 16.793 & 22.595 & 0.195 \\
\hline 30.235 & 520.000 & 5.815 & 23.775 & 37.140 & 1.325 & 178.428 & 196.333 & 6.763 & 57.210 & 281.380 & 0.280 \\
\hline-18.305 & 342.500 & 2.733 & 60.427 & 57.987 & 0.168 & -22.458 & 775.000 & 3.938 & 44.020 & 18.253 & 0.435 \\
\hline-3.257 & 215.000 & 5.028 & 10.725 & 62.240 & 0.130 & 0.220 & 642.500 & 9.513 & 49.735 & 49.795 & 1.305 \\
\hline 44.873 & 3500.000 & 51.660 & 17.143 & 13.005 & 0.460 & 6.305 & 750.000 & 6.115 & 30.088 & 27.445 & 0.240 \\
\hline-18.065 & 332.500 & 15.263 & 30.675 & -8.118 & 0.060 & -16.990 & 1369.000 & 2.658 & 42.238 & 15.055 & 1.058 \\
\hline-48.018 & 197.500 & 5.103 & 23.855 & 51.248 & -0.073 & -4.443 & 739.250 & 2.855 & 14.315 & 1.723 & 0.485 \\
\hline 25.350 & 1300.000 & 3.180 & 30.215 & -6.185 & 0.273 & -29.158 & 840.000 & 4.770 & 33.123 & -4.450 & -0.770 \\
\hline 2.608 & 362.500 & 2.948 & 40.620 & 2.095 & 1.180 & 1.243 & 275.000 & 5.250 & 9.443 & 12.208 & 1.163 \\
\hline-26.118 & 104.000 & 6.298 & -5.480 & 6.553 & 0.083 & -84.318 & 116.667 & 3.470 & 3.505 & 48.287 & 0.873 \\
\hline 45.248 & 432.500 & 6.903 & 25.160 & 39.855 & 0.550 & -0.783 & 295.500 & 2.755 & 10.935 & 9.225 & 0.255 \\
\hline 29.088 & 242.500 & 3.995 & -6.813 & 1.323 & -0.163 & -3.268 & 170.500 & 5.695 & 14.435 & 10.088 & 0.745 \\
\hline 15.455 & 420.000 & 3.590 & 433.520 & 6.175 & 0.343 & -8.405 & 76.500 & 3.805 & 73.783 & 40.838 & 1.293 \\
\hline 596.525 & 562.500 & 8.153 & 31.773 & 28.333 & 2.133 & 43.045 & 431.250 & 3.388 & 3.773 & 1.023 & 0.243 \\
\hline-0.508 & 587.750 & 5.473 & 20.638 & 33.588 & 1.243 & 71.525 & 71.250 & 6.063 & 24.575 & 102.623 & 0.295 \\
\hline 15.725 & 440.000 & 2.858 & 4.443 & 0.443 & 0.338 & 434.108 & 135.000 & 3.783 & 5.295 & 486.618 & 0.983 \\
\hline-5.027 & 328.250 & 3.410 & 18.400 & 16.175 & 0.313 & 3.497 & 105.000 & 5.190 & 32.937 & 38.383 & 0.723 \\
\hline-1.638 & 2800.000 & 5.000 & 27.255 & 1.100 & 1.043 & 9.613 & 247.500 & 4.713 & 33.693 & 25.765 & 0.238 \\
\hline-21.625 & 1650.000 & 6.350 & 52.975 & 39.735 & 0.985 & 8.333 & 215.000 & 9.205 & 1.503 & 9.003 & 0.765 \\
\hline
\end{tabular}

TABLE XII. THE RESULTS OF PRIORITIZING TOP AND ACTIVE COMPANIES IN STOCK EXCHANGE

\begin{tabular}{|c|c|c|c|c|c|c|c|c|c|c|c|}
\hline Rank & $C C_{i}$ & $d_{i}^{-}$ & $d_{i}^{*}$ & Rank & $C C_{i}$ & $d_{i}^{-}$ & $d_{i}^{*}$ & Rank & $C C_{i}$ & $d_{i}^{-}$ & $d_{i}^{*}$ \\
\hline 1 & 0.476 & 2.545 & 2.805 & 23 & 0.415 & 2.321 & 3.266 & 45 & 0.397 & 2.204 & 3.350 \\
\hline 2 & 0.471 & 2.492 & 2.797 & 24 & 0.415 & 2.287 & 3.225 & 46 & 0.397 & 2.099 & 3.191 \\
\hline 3 & 0.462 & 2.405 & 2.803 & 25 & 0.415 & 2.265 & 3.194 & 47 & 0.393 & 2.117 & 3.272 \\
\hline 4 & 0.447 & 2.337 & 2.897 & 26 & 0.415 & 2.294 & 3.237 & 48 & 0.390 & 2.098 & 3.281 \\
\hline 5 & 0.436 & 2.285 & 2.952 & 27 & 0.414 & 2.277 & 3.217 & 49 & 0.378 & 2.049 & 3.375 \\
\hline 6 & 0.431 & 2.389 & 3.155 & 28 & 0.414 & 2.284 & 3.232 & 50 & 0.370 & 1.853 & 3.157 \\
\hline 7 & 0.428 & 2.310 & 3.093 & 29 & 0.414 & 2.232 & 3.160 & & & & \\
\hline 8 & 0.427 & 2.232 & 2.999 & 30 & 0.410 & 2.194 & 3.162 & & & & \\
\hline 9 & 0.426 & 2.318 & 3.119 & 31 & 0.408 & 2.237 & 3.241 & & & & \\
\hline 10 & 0.426 & 2.303 & 3.103 & 32 & 0.408 & 2.188 & 3.174 & & & & \\
\hline 11 & 0.425 & 2.258 & 3.059 & 33 & 0.407 & 2.201 & 3.210 & & & & \\
\hline 12 & 0.424 & 2.330 & 3.160 & 34 & 0.406 & 2.171 & 3.170 & & & & \\
\hline 13 & 0.423 & 2.195 & 2.997 & 35 & 0.406 & 2.190 & 3.207 & & & & \\
\hline 14 & 0.422 & 2.237 & 3.067 & 36 & 0.405 & 2.247 & 3.297 & & & & \\
\hline 15 & 0.421 & 2.299 & 3.162 & 37 & 0.404 & 2.151 & 3.174 & & & & \\
\hline 16 & 0.420 & 2.263 & 3.128 & 38 & 0.404 & 2.061 & 3.044 & & & & \\
\hline 17 & 0.418 & 2.253 & 3.141 & 39 & 0.403 & 2.140 & 3.175 & & & & \\
\hline 18 & 0.418 & 2.247 & 3.133 & 40 & 0.401 & 2.126 & 3.170 & & & & \\
\hline 19 & 0.417 & 2.294 & 3.202 & 41 & 0.401 & 2.183 & 3.257 & & & & \\
\hline 20 & 0.417 & 2.231 & 3.114 & 42 & 0.401 & 2.155 & 3.215 & & & & \\
\hline 21 & 0.416 & 2.272 & 3.185 & 43 & 0.398 & 2.066 & 3.120 & & & & \\
\hline 22 & 0.416 & 2.275 & 3.196 & 44 & 0.397 & 2.145 & 3.252 & & & & \\
\hline
\end{tabular}




\section{DISCUSSION \& CONCLUSION}

Company stock ranking is a method to convert raw data into relevant information for decision-making. Ranking can be performed based on different parameters such as the criteria and fundamental analysis elements. The aim of this study is to provide a fuzzy multi-criteria decision making model to rank the stocks of companies of Tehran Stock Exchange. At first by using stock exchange experts as a decision-making group and then by using non-parametric statistical analysis to establish ranking criteria. Then, the ANP multi-fuzzy technique is used to determine their weight. Then, the fuzzy TOPSIS technique is used and the fifty top stocks in the Stock Exchange were ranked. After performing the ranking for sensitivity analysis of criteria weight, their weights are shifted mutually with each; hence, the rank of companies will be compared in fifty-one situations, by selecting two criteria of six criteria. A consistency is shown. The company that was placed in the second rank in main ranking occupied the first rank in all 51 situations and the company occupied the second rank in all 51 situations was placed in the first rank in main ranking. It is noteworthy that the decision maker can present a ranking by giving the desired amount of six indicators of the company. One can even begin to analyze the sensitivity of rate changes by criteria weight shift. The first ranking company has a higher special value per share and $\mathrm{D} / \mathrm{E}$ allocation ratio in comparison to other companies.. In the same manner, comparing financial ratios with other companies will uncover confirm acceptability and accuracy of ratings. Moreover, the results of interviews with the authorities on the final indicators of the research represent that the criteria are the most basic possible indicators for ranking stocks so that some experts believe that 80 percent of ranking result depends on three first factors that have more weights. In addition, the results of surveying experts about shares' obtained ranks in the ranking express that the results are very much close to reality; especially, companies with higher ranks can meet the demands of shareholders largely.

\section{SugGestions FOR Future StUdies}

Further studies can define subscales for each scale through more investigations; they can also solve the model according to mutual impacts of subscales of each group on each other as well as on other scales and subscales. As there are many problems in estimating the beta value in Tehran Stock Exchange, one can extract beta coefficient from other models like Dimson instead of using Rahavard Novin Software in order to obtain better results.

\section{REFERENCES}

[1] E. Onder, A. Hepsen, "Combining time series analysis and multi criteria decision making techniques for forecasting financial performance of banks in Turkey", International Conference on Applied Business and Economics (ICABE-13), pp. 530-555, October, 2013

[2] C. Kao, R. E. Steuer, "Value of information in portfolio selection, with a Taiwan stock market application illustration", European Journal of Operational Research, Vol. 253, No. 2, pp. 418-427, 2016

[3] K. Y. Shen, G. H. Tzeng, "Combined soft computing model for value stock selection based on fundamental analysis", Applied Soft Computing, Vol. 37, No. 1, pp. 142-155, 2015

[4] H. Ince, "Short term stock selection with case-based reasoning technique", Applied Soft Computing, Vol. 22, No. 2, pp. 205-212, 2014

[5] M. Amile, M. Sedaghat, M. Poorhossein, "Performance Evaluation of Banks using Fuzzy AHP and TOPSIS, Case study: State-owned Banks, Partially Private and Private Banks in Iran", Caspian Journal of Applied Sciences Research, Vol. 2, No. 3, pp. 10-15, 2013

[6] S. Ansari, M. Kazemi, A. Eshlaghy, "An application of fuzzy TOPSIS for ranking strategies”, Management Science Letters, Vol. 4, No. 4, pp. 663-668, 2014

[7] K. Y. Shen, M. R. Yan, G. H. Tzeng, "Combining VIKOR-DANP model for glamor stock selection and stock performance improvement", Knowledge-Based Systems, Vol. 58, No. 4, pp. 86-97, 2014

[8] X. Bi, R. A. Isaili, Q. Zheng, "Evaluation of Wastewater Treatment Quality in the West Bank-Palestine Based on Fuzzy Comprehensive Evaluation Method", 21st International Conference on Industrial Engineering and Engineering Management, pp. 219-221, 2015

[9] A. E. La Plante, J. C. Paradi, "Evaluation of bank branch growth potential using data envelopment analysis", Omega, Vol. 52, No. 2, pp. $33-41,2015$ 